\title{
Optomechatronic Technology: The Characteristics and Perspectives
}

\author{
Hyungsuck Cho, Senior Member, IEEE, and Min Young Kim
}

\begin{abstract}
In recent years, optical technology has been incorporated into mechatronic systems at an accelerated rate, and as a result, a great number of machines/systems with smart optical components have been introduced. This integrated technology is termed "optomechatronics." This paper introduces the fundamental concept, definition, and characteristics of the technology by analyzing the characteristics of a variety of practical optomechatronic systems. The introduction describes how optical and mechatronic components are physically coupled to each other to form optomechatronic integration. With this observation, we describe the nature and integration concept of the technology, from which we can derive the technology-driven fundamental functionalities in some detail. Based upon the knowledge on basic optomechatronic integration and functions, we analyze optomechatronic systems in general from the viewpoint of system configuration and design and, thus, the roles of optical technology in overall system performance being learned and the synergistic effects due to its fusion with mechatronics being understood.
\end{abstract}

Index Terms-Functional modules, mechatronics, optoelectronics, optomechanics, optomechatronic integration, technology fusion.

\section{INTRODUCTION}

$\mathbf{T}$ HE characteristics of modern engineered products, machines, processes, or systems are that they are configured in somewhat complicated, multidisciplinary fashion and that most of them have moving parts requiring control of their mechanical or dynamic constructions to achieve a desired performance. This necessitates the interaction between various technologies such as mechanism, sensor, actuator, control, signal processing, microprocessor, optics, software, communication, and so on. This trend will be accelerated in the future, since the multidisciplinary integration approach has been found to enhance their performance and yield synergistic effects, otherwise unavailable. Looking back into the past two decades, we have witnessed a technological evolution that has led to advancements in machines, processes and systems. In particular, mechatronic technology integrated by mechanical, electronic, and computer technologies significantly contributed to this evolution. However, mechatronic technology alone seems to have

Manuscript received October 23, 2003; revised December 8, 2004. Abstract published on the Internet April 28, 2005.

H. S. Cho is with the Laboratory for Vision System and Machine Intelligence, Department of Mechanical Engineering, Korea Advanced Institute of Science and Technology, Taejon 305-701, Korea (e-mail: hscho@lca.kaist.ac.kr).

M. Y. Kim is with the Surface Mounting Technology Department, Mirae Corporation, Cheonan 330-220, Korea (e-mail: mykim@mirae.com).

Digital Object Identifier 10.1109/TIE.2005.851689

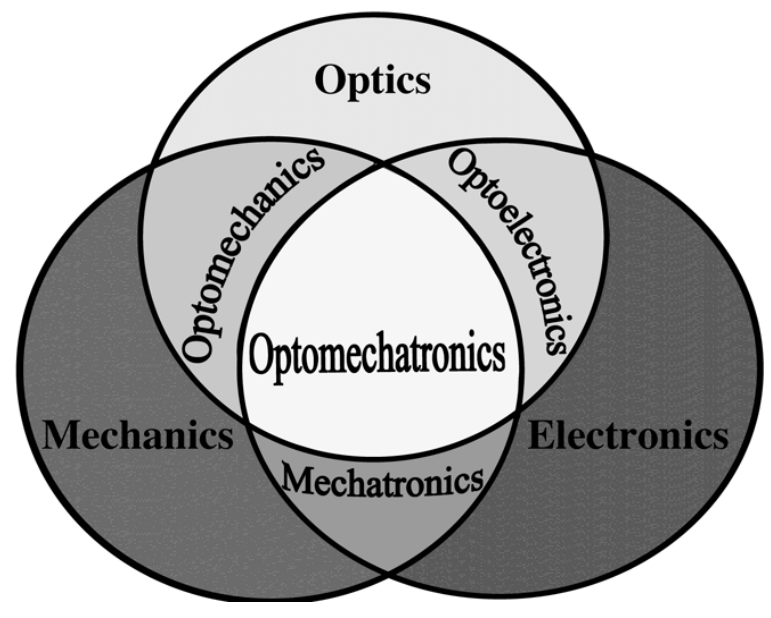

Fig. 1. Definition of optomechatronic technology [9].

limitations in achieving increased flexibility and intelligence and high functionality.

In recent years, optical elements have been increasingly incorporated at an accelerated rate into mechatronic systems, and vice versa [1]-[8]. This may be attributable to the fact that optically integrated technology provides solutions to complex problems by achieving a desirable function or performance that mechatronic technology alone cannot provide. As a result, mechatronic or optical products, machines, and systems have further evolved toward a state of precision, reduced size, and greater intelligence and autonomy. In the future, this trend will continue to map out the direction of next-generation technologies associated with mechatronic and optical engineering-related fields. The technology fusion in this new paradigm is termed as "optomechatronics" or "optomechatronic technology," which is an integration of optical and mechatronic technologies, as shown in Fig. 1. The fused technology in the shaded area is certainly multifaceted in nature and thus requires the interaction between the fundamentals of technologies relevant to form the optomechatronics. Despite this nature, however, little effort to systematically treat and analyze this engineering field has been made, to date, to exploit it further.

This paper, therefore, has two major objectives. The first is to introduce the technical field "optomechatronics" by presenting the definition, fundamentals and application aspects of the technology. The second is to provide an integrated view of optomechatronics, and thereby help us understand how optical elements can be integrated with mechatronic elements to produce certain functionalities. An attempt is made to analytically represent and classify optomechatronic systems depending 


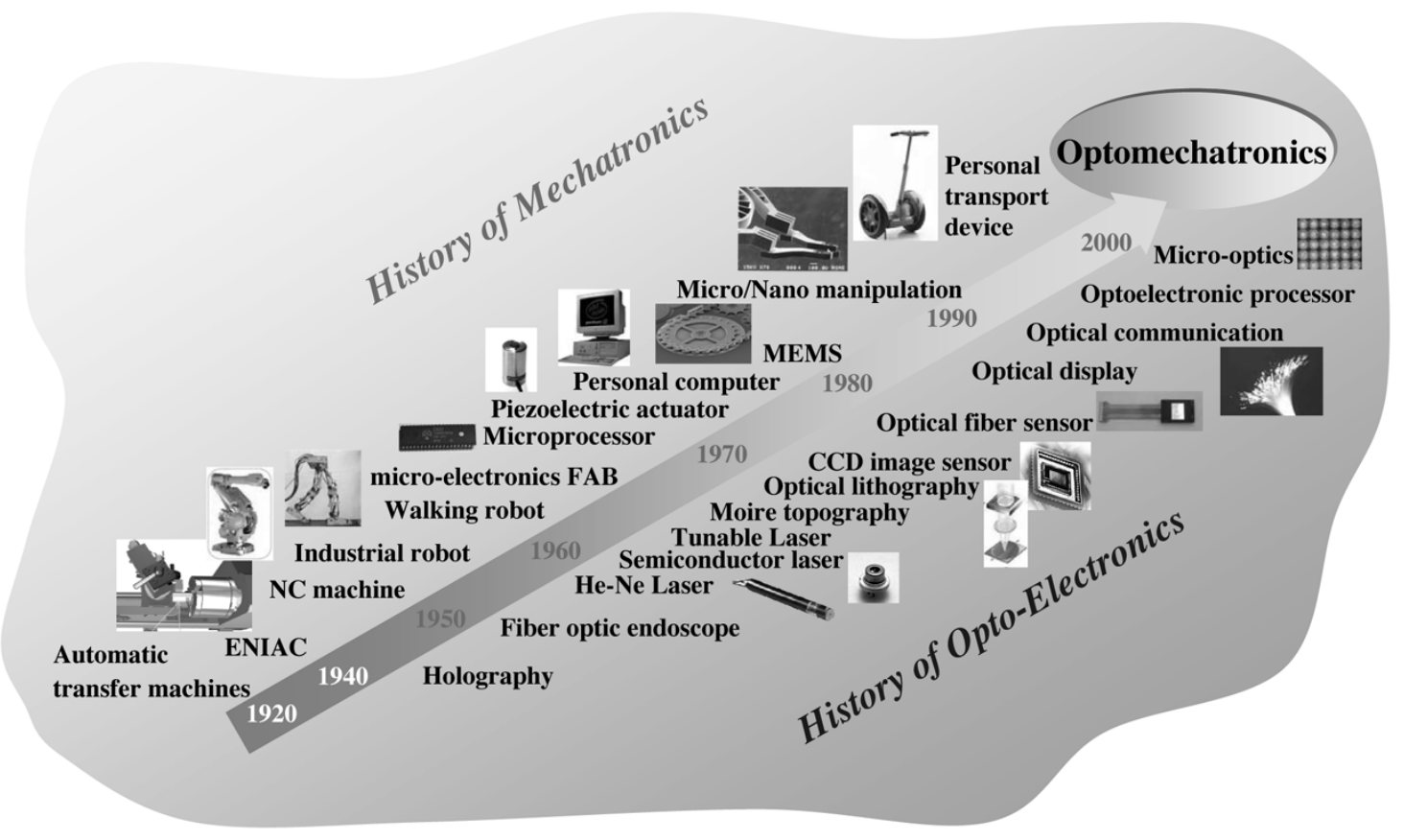

Fig. 2. History of optomechatronics [9].

on the types of integration. Also, optomechatronics-generated fundamental functionalities, optomechatronic design considerations and synergistic effects and future perspectives of the technology are discussed in some detail.

\section{OPTOMECHATRONIC INTEGRATION: HSITORICAL BACKGROUND AND TECHNOLOGY EVOLUTION}

Optomechatronics has its roots in technological developments of mechatronics and optoelectronics. Fig. 2 shows the chronology of those developments [9]. In the 1960s, the electronic revolution came with the integration of transistor and other semiconductor devices into monolithic circuits and, in 1971, semiconductor fabrication technology brought about a tremendous impact on a broad spectrum of technological fields. In particular, the development created a synergistic fusion of hardware and software technologies by combining a variety of technologies and computer technology. In the 1980s, the semiconductor technology also created microelectromechanical systems (MEMS), and this brought about a new dimension of machines/systems, microsizing their dimension.

Along with this evolution, optoelectronic integration has continued over the last 40 years, since the advent of the laser in 1960. This was made possible with the aid of advanced fabrication methods such as chemical vapor deposition, molecular-beam epitaxy, and focused-ion-beam micromachining. The charge-coupled device (CCD) image sensor developed in 1974 not only originated computer vision technology but also, starting in 1976, opened up a new era of optical technology and optical fiber sensors. In recent years, microoptics has been developed, incorporating with MEMS technology, and becoming a core technology of microoptoelectro mechanical system (MOEMS). The developed optical components and devices possessed a number of favorable characteristics. These components did not involve contact, were noninvasive, were easy to transduce, had a wide sensing range, were insensitive to electrical noises, had distributed sensing and communication, and had high bandwidth.

Naturally, optical elements exhibiting these favorable characteristics began to be integrated with those of mechatronic elements. When a system or machine is integrated in this way, i.e., optically, mechanically, and electronically, it is called an optomechatronic system. Fig. 3 [16]-[18] illustrates some of practical optomechatronic systems currently being used that evolved from their original old version, and Table I summarizes evolutions of representative products. The functions of the chip mounter which originally were mostly mechanical, are now being carried out by the integrated devices which include part position estimator, visual sensors, and servo control unit. The projector being used as a data representation device is now in a transitional state from the CRT type to liquid crystal display (LCD) or digital light projector (DLP) as shown in Fig. 3(b). In particular, DLP is a typical optomechatronic system in which optical switching steers and projects the light beam into a screen. The mobile robot shown in Fig. 3(c) now replaces some human intelligence (perception and pattern recognition) by optomechatronic systems which can perceive navigation environment, and recognize the environment based upon the perception.

In the next section, to provide better understanding of and insight into this technology we will briefly illustrate a variety of optomechatronic systems being used in practice, and review the basic roles of optical technology in these systems.

\section{UNDERSTANDING OPTOMECHATRONIC SYSTEMS}

\section{A. Basic Roles of Optical Elements}

The major functions and roles of optical elements in optomechatronic systems can be categorized into several technological domains: illumination, sensing, actuating, data 


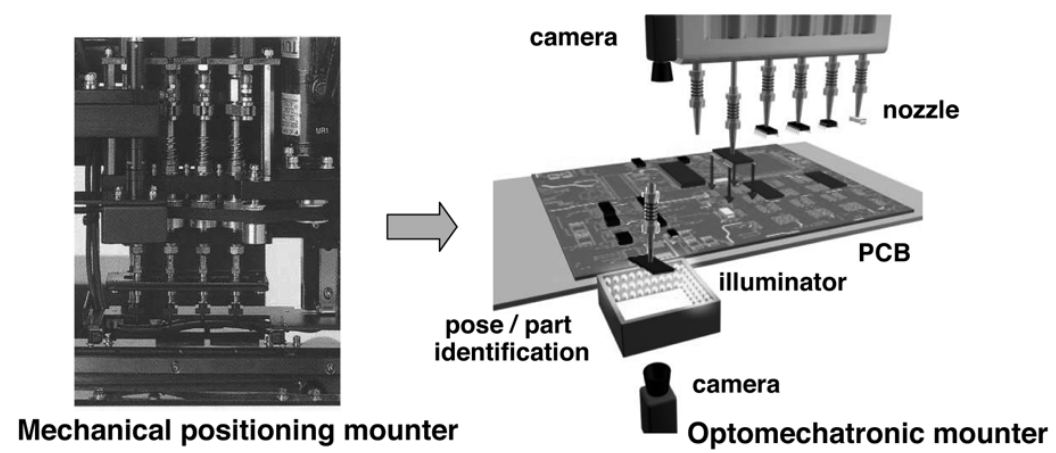

(a) Surface mounting device (SMD)
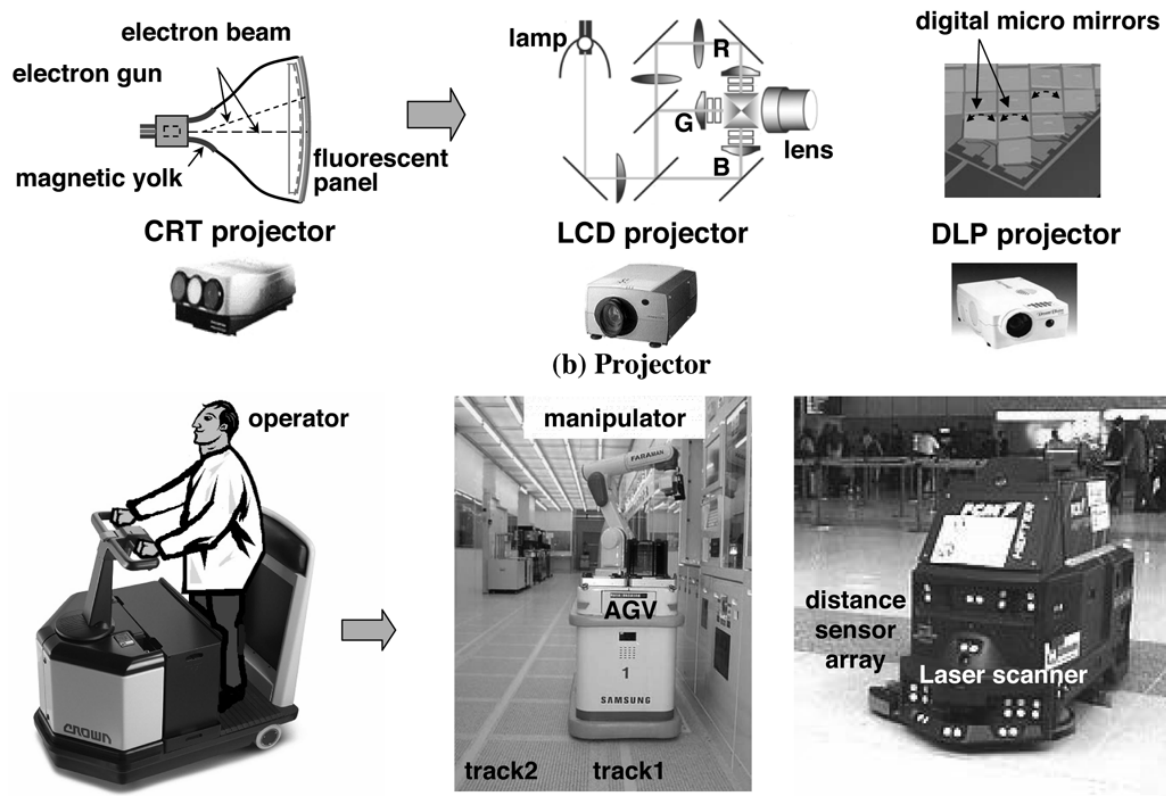

(b) Projector

Human guided vehicle [16]

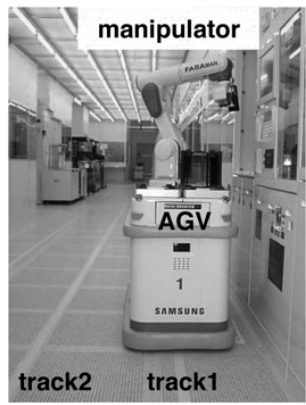

Automatically guided robot [17]

(c) Mobile robot

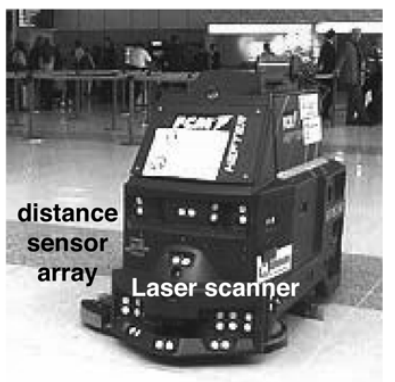

Free ranging mobile robot for cleaning [18]

Fig. 3. Evolutionary trend in various practical systems.

TABLE I

EVOLUTION IN VARIOUS PRODUCTS

\begin{tabular}{l|l}
\hline technology / product & \multicolumn{1}{|c}{ technological trend } \\
\hline data storage disk & mechanical recording $\rightarrow$ magnetic recording ' optical recording \\
\hline printer & dot printer $\rightarrow$ thermal printer / ink jet printer $\rightarrow$ laser printer \\
\hline projector & CRT $\rightarrow$ LCD $\cdot$ DLP projector $\rightarrow$ TFT- LCD , PDP, DMD \\
\hline IC chip mounter & $\begin{array}{l}\text { partially manual automated assembly } \rightarrow \text { mechanically } \\
\text { automated assembly } \rightarrow \text { assembly with visual chip recognition }\end{array}$ \\
\hline PCB inspection & naked eye inspection $\rightarrow$ optical / visual inspection \\
\hline camera & $\begin{array}{l}\text { manual film camera } \rightarrow \text { motorized zoom, auto exposure, auto } \\
\text { focusing } \rightarrow \text { digital camera (CMOS, CCD device })\end{array}$ \\
\hline $\begin{array}{l}\text { coordinate } \\
\text { measuring machine } \\
(\text { CMM })\end{array}$ & $\begin{array}{l}\text { touch probe } \rightarrow \text { optical probe } \rightarrow \text { touch probe }+ \text { visual / optical } \\
\text { sensing }\end{array}$ \\
\hline
\end{tabular}

(signal) storage, data transmitting, data displaying, computing, and material property variation.
Optical technologies can provide the source of photometric radiant energy incident to object surfaces. In addition, optical sensors provide fundamental information on physical quantities such as force, temperature, pressure, and strain as well as geometric quantities such as angle, velocity, etc. The actuating function is implemented from the characteristics that light can change physical properties of materials by increasing the temperature of the material or affecting electrical environment. In the case of "data storage," optics help the digitized data composed of 0's and 1's to be stored in media and read optically. Additionally, light can be used as a good medium for delivering data. In optical communication, the digitized raw data, such as text or a picture, are transformed into a light signal, delivered to the other side of the optical fiber, and decoded as the raw data. Next, optical technologies can provide the function to transfer some information to users in the form of an image or graph. Various displaying devices implemented by optical technologies have been developed, which all are made of pixel elements composed of three basic cells that emit or transmit red, green, and blue light. Optical computing performed by using optical switches, gates, and flip-flops in their logic operation is 
a promising function; for example, optical devices can switch states in about a picosecond or one-thousandth of one-billionth of a second. Lastly, optical technology generates energy which can be used for material property variation. Various laser-assisted material processing methods utilize a laser beam as the energy input.

\section{B. Basic Roles of Mechatronic Elements}

The major functions and roles of mechatronic elements in optomechatronic systems can be categorized into the following five technological domains [10]: sensing, actuation, information feedback, motion/state control, and embedded intelligence with microprocessor.

First, the transducer technology used for sensing nowadays enjoys the integrated nature of mechatronics. The majority of sensors belong to this category. Second, the drivers and actuators produce a physical effect such as a mechanical movement or a change of property and condition. Third, one of the critical functions of the mechatronics is to feed back information for certain objectives. Fourth, the control of motion or state of systems is a basic functionality that can be provided by mechatronics. Lastly, mechatronic systems implemented with microprocessors provide many important functions, for example, the stored/programmed control, the digital signal processing, and the design flexibility for the whole system. In addition, advantages of the integration within a small space and the low power consumption are attractive features.

\section{Practical Optomechatronic Systems: An Illustration}

Examples of optomechatronic systems are found in a number of control and instrumentation, inspection and test, optical, manufacturing, consumer and industrial electronics, MEMS, automotive, bio-applications, and many other fields of engineering. Let us illustrate some such applications to provide a better understanding of optomechatronics.

A modern digital camera is a typical device that is operated by optomechatronic components. This optomechatronic configuration provides an aperture control, a shutter zoom control, and a focusing adjustment together with an illuminometer designed to perform well regardless of the ambient brightness change, as shown in Fig. 4(a).

An atomic force microscope (AFM) is composed of several optomechatronic components; a cantilever probe, a laser source, a position sensitive detector (PSD), a $z$-axis piezoelectric actuator and a servo controller, and an $x-y$ servoing stage as shown in Fig. 4(b) [1]. In the case of a constant-force mode the deflection of the cantilever is used as input to a feedback controller, which, in turn, moves the piezoelectric element up and down in the $z$ direction, responding to the surface topography by holding the cantilever deflection constant.

An optical disk drive is an optomechatronic system as shown in Fig. 4(c). The disk is composed of an optical head that carries a laser diode, a beam focus servo that dynamically maintains the laser beam in focus and a fine track servo that accurately positions the head at a desired track. Nowadays, a washing machine effectively utilizes optoelectronic components to improve washing performance. It has an ability to feedback control the water temperature within the washing drum and adjust washing cycle time, depending upon the dirtiness of the inside washing water area [2]. As shown in Fig. 4(d), the machine is equipped with an optomechatronic component to achieve such function.

An optically ignited mechatronic weapon system is shown in Fig. 4(e). The system has a laser ignition system mounted in the breech of a gun and this was the first major change in the way that guns are fired in the past 100 years [3]. The system replaces the conventional firing method that has utilized an ignite material to ignite the propellant charge.

Optical microelectromechanical (MEM) components are miniature mechanical devices capable of moving and directing light beam as shown in Fig. 4(f). In optical networks, optical MEMs can dynamically attenuate switch, compensate, combine and separate signals, all optically [4].

Fig. 4(g), illustrates a fine image fiberscope device which can perform active curvature operations for inspection of tiny and confined areas such as a microfactory [5]. A shape memory alloy (SMA) coil actuator enables the fiberscope to move through a tightly curved area. A number of manufacturing processes require feedback control of in-process state information that must be detected by optoelectronic measurement systems. One such process, illustrated in Fig. 4(h), is a welding system equipped with an optical sensory system composed of a laser triangulation sensor for seam tracking and a noncontacting IR optical temperature sensor for weld quality estimation [11], [12].

\section{OPTOMECHATRONIC INTEGRATION}

Based upon what we have previously observed from various optomechatronic systems, we can summarize the following characteristic points: 1) they possess one or more functionalities to carry out certain given tasks; 2) to produce such functionalities, several basic functional modules are required to be appropriately combined (combining such functional modules in a desired manner can be achieved by converting or manipulating one signal to another in a desired form, using the basic mechanical, optical, or electrical signal); and 3) optomechatronic systems are hierarchically composed of subsystems, which are then composed of units or components. In other words, elements, components, units, or subsystems are integrated to form an optomechatronic system.

The characteristics due to integration mentioned in the above can allow us to lay the foundations to derive and create the concept of optomechatronic technology. To begin with, we consider basic function modules that can produce or create fundamental functionalities. A functional module is defined by the module that is a basis to produce a basic function. The functional modules include transformation, manipulation, sensing, control, transmission, and display of the signals which are the physical variables originating from three engineering domains. It can be shown that two modules, transformation and manipulation, can be properly combined to represent the rest of the functional modules. To take an example, let us suppose that a beam scanner operated by an electric motor interacts with a light beam from a laser source and diverts the incident beam to a desired direction. This scanned beam is then detected by a photodetector. 


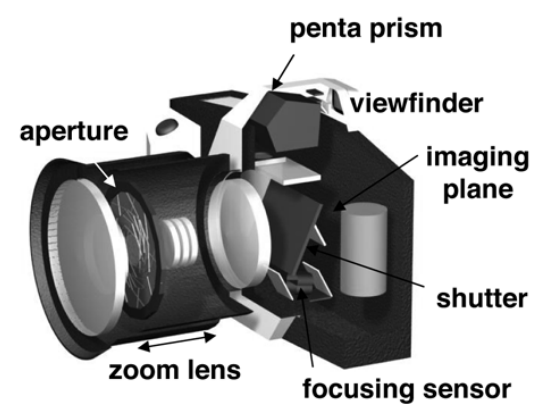

(a) Digital camera

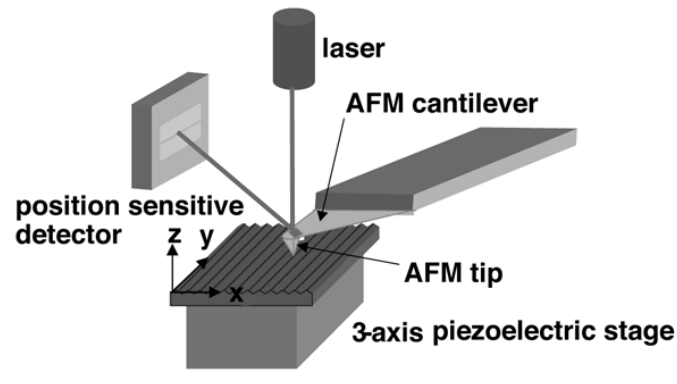

(b) Atomic force microscope (AFM)

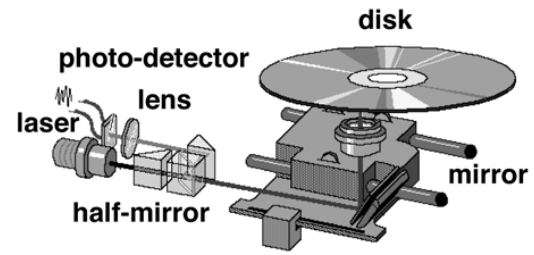

(c) Optical storage disk

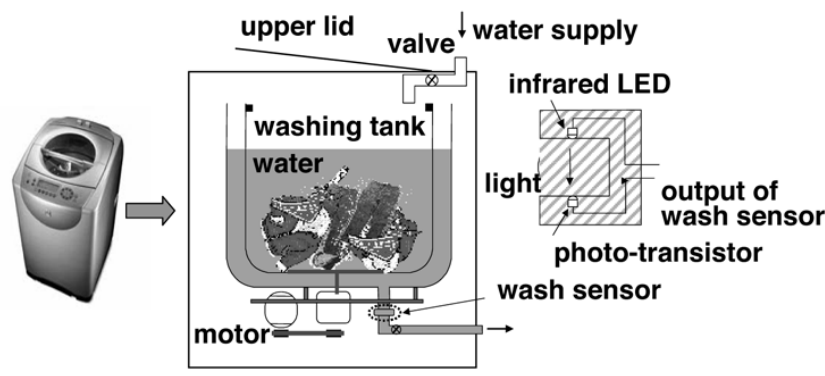

(d) Modern washing machine with optical sensory feedback

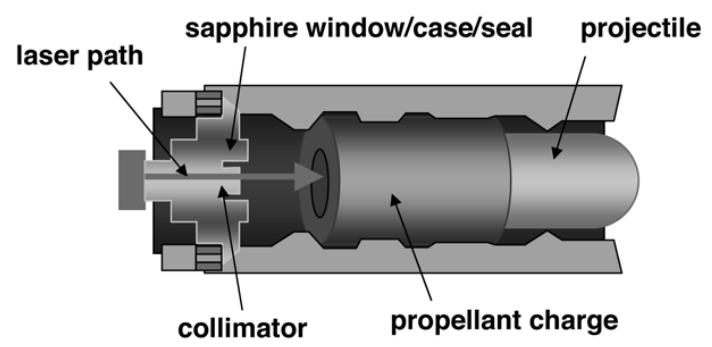

(e) Optically ignited mechatronic weapon system

image guide fiber

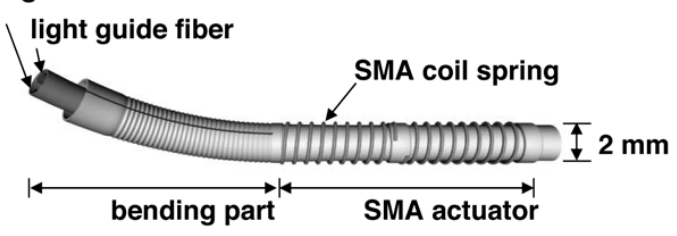

(g) Fiber scope device for inspection for micro factory

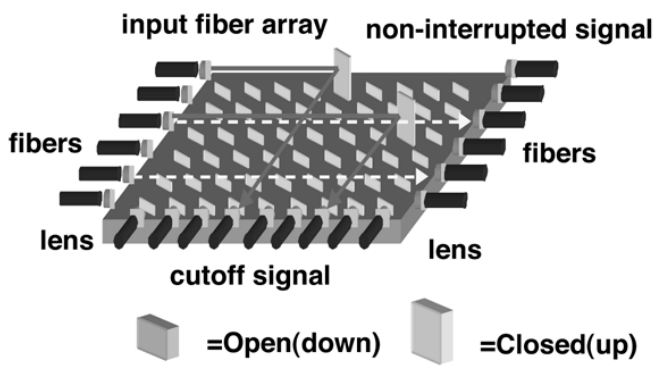

(f) $\mathbf{n} \times \mathbf{n}$ optical switching system

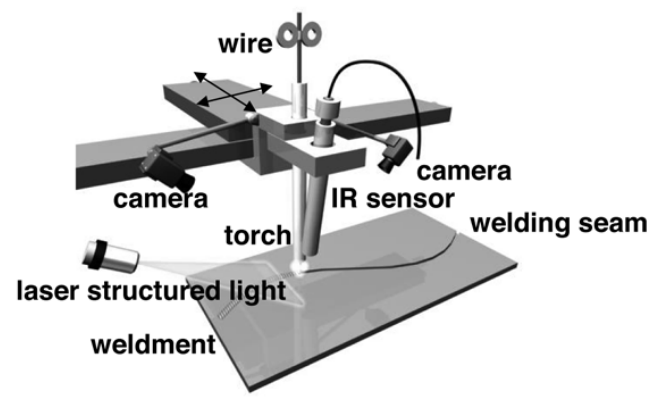

(h) Welding system with monitoring and control units

Fig. 4. Typical examples of optomechatronic systems.

The whole scanning procedure can be conveniently represented in a symbolic form by using the basic modules as follows:

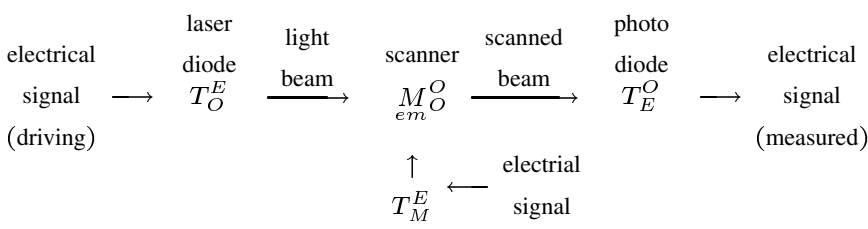

where $T_{O}^{E}, T_{E}^{O}$, and $T_{M}^{E}$ indicate the transformation from electrical to optical, from optical to electrical and from electrical to mechanical, respectively, and $M_{O}^{O}$ indicates a signal manipulator that modulates laser beam by electromechanical means. From this above symbolic representation we can see that: 1) this representation method can be extended to most optomechatronic integration processes and 2) the basic modules are composed of the transformation and manipulation modules $T$ and $M$. To this end, we will consider here only two basic functional modules, transformation and manipulation modules.

The signal transformation is the module that converts signals from one form to another as summarized in Table II [13]. 
TABLE II

BASIC SIGNAL (ENERGY) TRANSFORMATIONS USED FOR OPTOMECHATRONIC INTEGRATION

\begin{tabular}{l|l|l}
\hline \multicolumn{1}{c|}{ Transformation } & \multicolumn{1}{c|}{ Phenomenon } & \multicolumn{1}{c}{ Typical device } \\
\hline optical $\rightarrow T_{E}^{O} \rightarrow$ electrical & $\begin{array}{l}\text { photovoltaic pyroelectric } \\
\text { photo emissive }\end{array}$ & photo diode \\
\hline electrical $\rightarrow T_{O}^{E} \rightarrow$ optical & emission of photon & light emitting diode \\
\hline optical $\rightarrow T_{M}^{O} \rightarrow$ mechanical & $\begin{array}{l}\text { photovoltaic } \\
\text { phase transformation }\end{array}$ & $\begin{array}{l}\text { optical actuator shape } \\
\text { memory actuator }\end{array}$ \\
\hline mechanical $\rightarrow T_{O}^{M} \rightarrow$ optical & radiation & black body \\
\hline electrical $\rightarrow T_{M}^{E} \rightarrow$ mechanical & $\begin{array}{l}\text { electromagnetism } \\
\text { piezo electric }\end{array}$ & $\begin{array}{l}\text { electric motor, } \\
\text { piezo actuator }\end{array}$ \\
\hline mechanical $\rightarrow T_{E}^{M} \rightarrow$ electrical & $\begin{array}{l}\text { induction } \\
\text { piezo electric }\end{array}$ & $\begin{array}{l}\text { electric generator, } \\
\text { piezo sensor }\end{array}$ \\
\hline
\end{tabular}

The first transduction type, optical-to-electrical $\left(T_{E}^{O}\right)$, produces electricity from optical input which can be derived from several phenomena such as photovoltaic phenomenon. The second type, electrical-to optical $\left(T_{O}^{E}\right)$, is the reverse case of the first and produces light from electric input. This type can be used for either light source or signal conversion for transmission. The third category of the transformation is the optical-to-mechanical $\left(T_{M}^{O}\right)$ form which yields mechanical signals from optical signals. This can be found from optical actuators which utilize some physical phenomena, such as inverse piezoelectric and material phase transformation. The fourth one with mechanical-to-optical form $\left(T_{O}^{M}\right)$ can be found from light radiated from hot bodies. The fifth one with electrical-to-mechanical form $\left(T_{M}^{E}\right)$ is commonly used in most mechatronic devices including motion-generating devices such as electric motors and piezoactuators. The last transformation type $\left(T_{E}^{M}\right)$ produces an electrical signal from a mechanical signal from phenomena such as several electric induction and piezoelectricity.

The signal manipulation is the module that diverts or modulates signals in a desired form. In Table III, typical signal manipulation methods are summarized in view of the basic operation principle and type of component involved. $M_{\mathrm{e}}^{O}$ and $M_{O}^{O}$ indicated in the table denote the manipulation of an optical beam by mechatronic means and that by electrical means, respectively. A variety of cases that can illustrate this mode can be found from a practical system such as mechanical scanner by which a laser beam is diverted in a regulated manner depending upon applications.

Next, let us consider the anatomy of such integration as depicted in Fig. 5. As can be seen from the figure, three basic optical, mechanical, and electronic elements are the basis of the integration. These elements are assembled together to constitute a component. The resulting components are being grouped into three which represent the integration of dissimilar elements, mechatronic $(M E)$, optomechanic $(O M)$, and optoelectronic $(O E)$. It is noted that the arrows indicate the integration described in the above. Up to this level, there occurs integration of optical and mechatronic elements. In unit level, however, we can see that there are seven types of such integration, namely, a combination of components such as optoelectronic
TABLE III

TyPES OF OPTICAL SIGNAL MANIPULATION

\begin{tabular}{|c|c|c|}
\hline beam manipulation & basic principle & type \\
\hline acousto-optical modulator & Bragg deflection & $M_{e m}^{o}$ \\
\hline mechanical chopper & beam chopping by blade & $M_{e m}^{o}$ \\
\hline photo-elastic modulator & changes in refraction index & $M_{e}^{o}$ \\
\hline electro-optical modulator & changes in refraction index & $M_{e}^{o}$ \\
\hline mechanical scanner & reflection & $M_{\mathrm{em}}^{o} \mathrm{o}$ \\
\hline acousto-optic scanner & Bragg deflection & $M_{e m}^{o}$ \\
\hline electro-optical scanner & reflection & $M_{e}^{o}$ \\
\hline
\end{tabular}

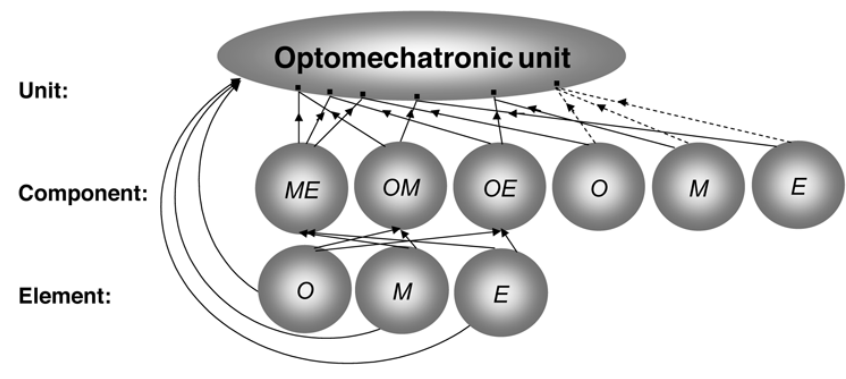

Fig. 5. Basic integration for optomechatronic technology.

plus mechatronic $(O E+M E)$, optomechanical plus mechatronic $(O M+M E)$, optomechanical plus optoelectronic $(O M+O E)$, optical plus mechatronic $(O+M E)$, optomechanical plus electronic $(O M+E)$, mechanical plus optoelectronic $(M+O E)$, and finally, a combination of three elements $(O+M+E)$. A variety of practical examples of this integration concept are illustrated in Fig. 6.

In summary, these signal transformation and manipulation modules together with seven integration types in the above are the core concepts making us certain that optical and mechatronic elements can be properly combined to create a variety of optomechatronic integrations, depending on applications.

\section{FUndAMENTAL FunCtions GenERATED VIA OPTOMECHATRONIC INTEGRATION}

A number of the fundamental functionalities can be obtained by the above integration. Indeed, a combination of six functional modules can produce a variety of unique functionalities. Taking just one example the intensity and directivity of illumination can be made changeable by optomechatronic integration and this functionality represents an important optical property. When viewing creation of functionality with this concept, we can list some of the functionalities that can be generated by the optomechatronic integration. 


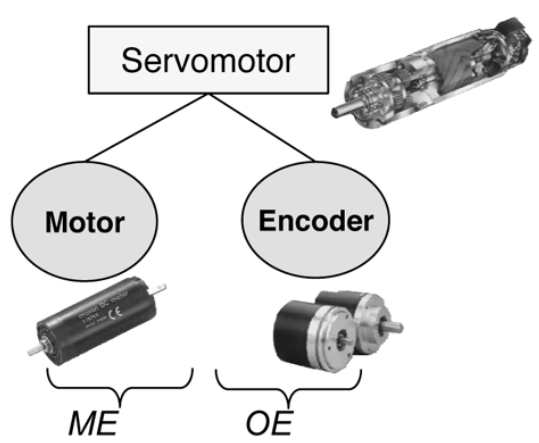

(a) $\mathrm{ME}+\mathrm{OE}$

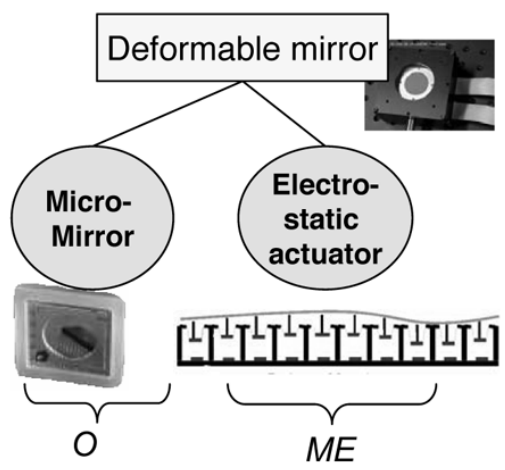

(c) $\mathrm{O}+\mathrm{ME}$

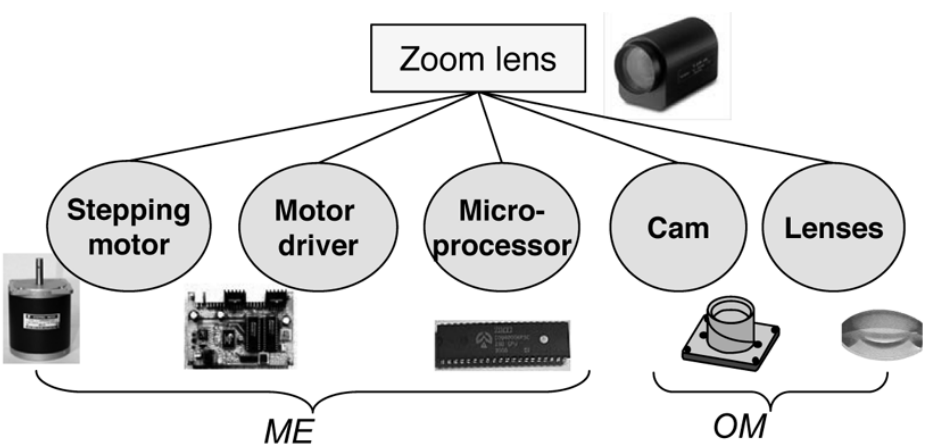

(b) $M E+O M$

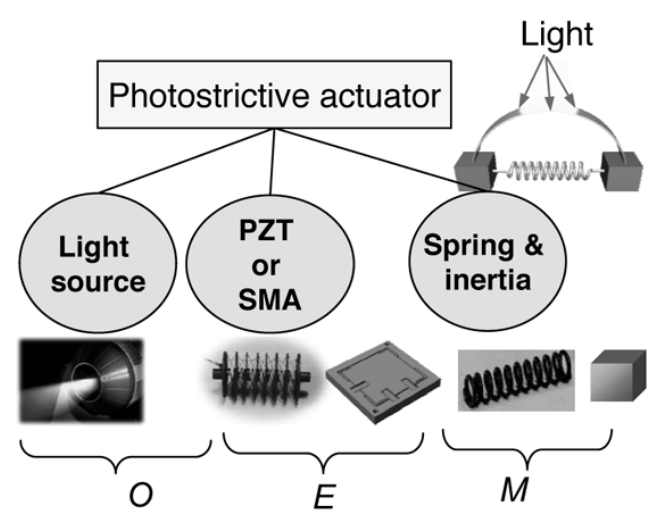

(d) $O+E+M$

Fig. 6. Illustrations of fused unit.

- Illumination control: Illumination needs to be adjusted depending on optical surface characteristics and surface geometry of objects in order to obtain a good quality image.

- Sensing: A number of sensors based on a principle of optomechatronic technology are used to make measurements of various types of physical quantities such as displacement, geometry, force, pressure, target motion, 3-D object shape, etc.

- Actuation: Two types of optomechatronic actuating principles can be listed. One typical type includes servomotors (electrical/hydraulic/pneumatic) into which optical sensors are embedded to measure their movement. The other type is the actuators generating a motion in purely optical principle.

- Optical scanning: Optical scanning action is provided by polygon mirror, galvanometer, acoustooptic deflector, active optical fiber scanners, and pan-tilt devices with a servoing mechanism embedded with optical sensors such as CCD cameras, fiber displacement sensors, etc.

- Optical feedback control: Visual/optical information is useful for the control of machines, processes, and systems. A number of mechatronic systems require this type of information feedback control.

- Optical data storage/retrieval: Data storage/retrieval is performed by a spinning optical disk in which the main functions are beam focusing and track following.

- Data switching: In all optical switching configurations, the system consists of a mirror, an actuator, a collimating lens, and input/output fibers through which light comes in, and is collimated. Then, by switching action, light is directed or switched from one fiber to another [14].

- Data display: Digital micromirror devices (DMD) [8] make projection displays by converting white-light illumination into full-color images via spatial light modulators with independently addressable pixels.

- Optical property variation: Optical properties such as polarization mode amplitude, deflection angle, and wave frequency of light beam can be varied according to the movement of a mechatronic element. A number of modulation devices adopt this principle.

- Sensor-based optical system control: In many cases, optical or visual systems are operated based on the information provided by external sensory feedback. Typical cases of this category are the visual/optical systems that react to sensory information such as tactile, force, displacement, velocity, acoustic sound, etc.

- Online optical pattern recognition: To recognize targets, pattern recognition is needed which usually employs optical correlation processing technique. In the case where robots try to find targets in unknown environments, or machines try to locate targets in carrying out tasks, recognizing and locating them are required in a real-time manner.

- Material processing: Material processing can be achieved by the integration of a laser optical source and mechatronic servomechanism. The system produces material property change or cut surface and heat-treat surfaces of work pieces. 


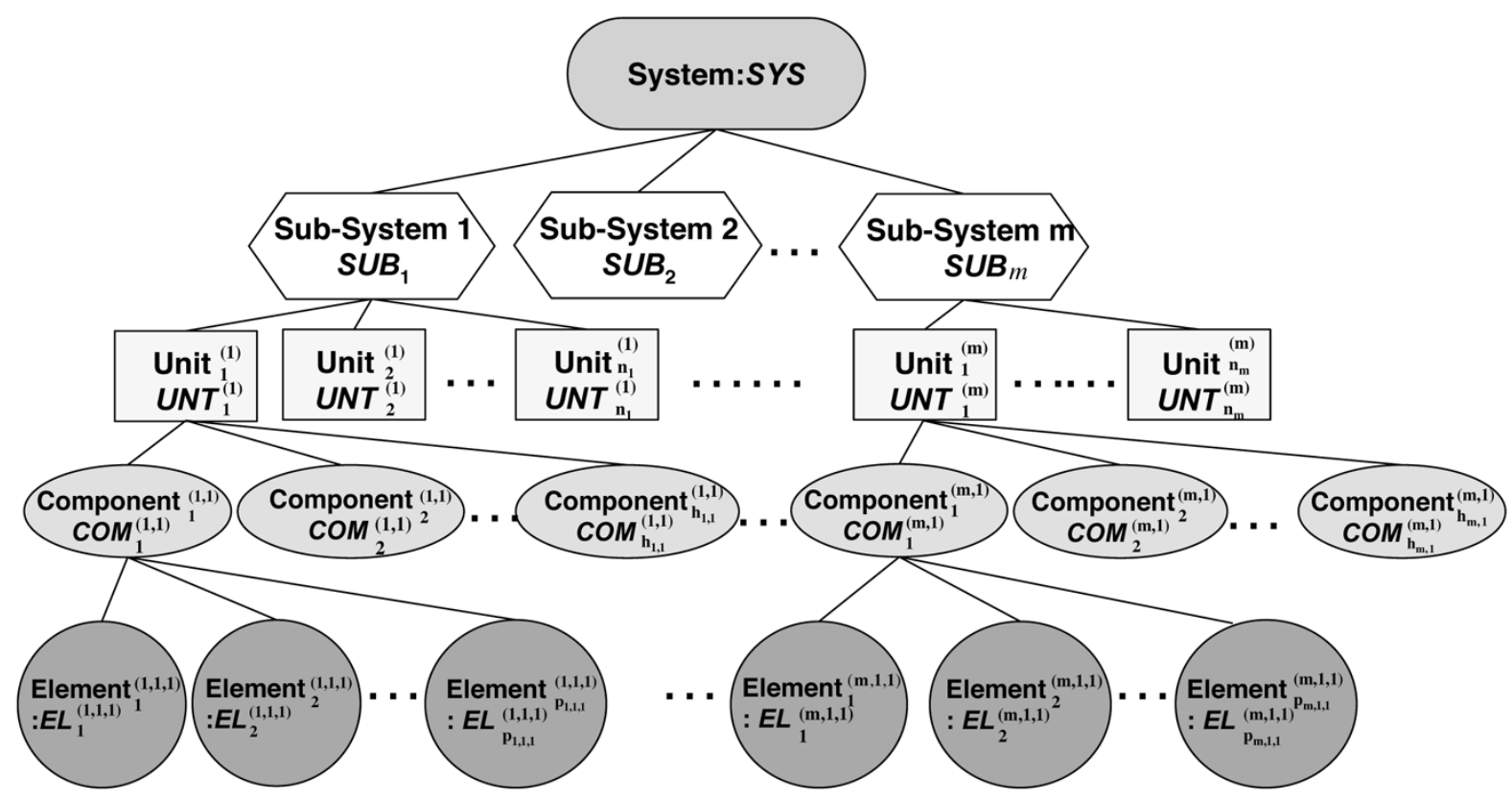

Fig. 7. A structure of optomechatronic system.

- Data transmission: Optical data transmission is widely used when data/signals obtained from sensors are subject to external electrical noise, when the amount of data needed to be sent is vast, or when operation is being done at a remote site.

\section{OPTOMECHATRONIC SYSTEM CHARACTERISTICS}

\section{A. Definition of Optomechatronic System}

Part of the functionalities discussed in the previous section may be produced by an optomechatronic unit, according to the definitions shown in Fig. 5. However, other parts of the functionalities requires more units in order to be properly represented. In this case, an integrated state called a "subsystem" is necessary. Therefore, in general, an optomechatronic system configured with a variety of units and subsystems in such a way that it creates certain functionalities in a desired manner. Viewing an optomechatronic system from this point of view, let us first define the optomechatronic system, and then classify the types of systems. Referring to Fig. 1, we assume that a system Sys encompasses seven different system groups associated with an optomechatronic system: mechanical system $M$; optical system $O$; electronic system $E$; optomechanical system $O M$; optoelectronic system $O E$; mechatronic system $M E$; and optomechatronic system $O P M E$. The optomechatronic system contains two mixed systems. One is the mechatronic system embedded with optical constituents denoted by $M E O$, and the other is the optical system embedded with mechatronic constituents denoted by $O E M$. Utilizing the concept of sets theory, these can be conveniently represented by the following definitions.

Definition 1: If a pure mechanical system, a pure optical system, and a pure electronic system are denoted, respectively, by $M_{p}, O_{p}$, and $E_{p}$, they are given by

$$
\begin{aligned}
M_{p} & =M \cap O^{C} \cap E^{C} \\
O_{p} & =O \cap M^{C} \cap E^{C} \\
E_{p} & =E \cap M^{C} \cap O^{C} .
\end{aligned}
$$

The above definition states that, for example, $M_{p}$ is the intersection of set $M$, complement of set $O$ denoted by $O^{C}$, and complement of set $E$ denoted by $E^{C}$.

Definition 2: If an optomechanical system, an optoelectronic system, and a mechatronic system are denoted, respectively, by $O M, O E$, and $M E$, they are given by

$$
O M=O \cap M \quad O E=O \cap E \quad M E=M \cap E .
$$

The above definition states that $O M, O E$, and $M E$ are the intersection of set $O$ and set $M$, the intersection of set $O$ and set $E$, and the intersection of set $M$ and set $E$, respectively.

Definition 3: The optomechatronic system $(O P M E)$ is the intersection of three sets $O M, O E$, and $M E$, that is, the set of all elements which belong to $O M, O E$, and $M E$

$$
O P M E=O M \cap O E \cap M E
$$

or

$$
O P M E=M E O \cap O E M .
$$

This definition states that $O P M E$ is the union of set $M E O$ and set $O E M$. The above three definitions can be similarly applied to optomechatronic units and subsystems.

\section{B. Optomechatronic System Architecture}

A system is assumed to be hierarchically structured and composed of elements, components, units, and subsystems as shown in Fig. 7. It is noted here that an element is the elementary part in the lowest level that is functionally not separable into another. Each subsystem may have its own hierarchical structure composed of subsystem layers in different levels, but here, to simplify notation, we will assume all subsystems have a single-layer structure. 


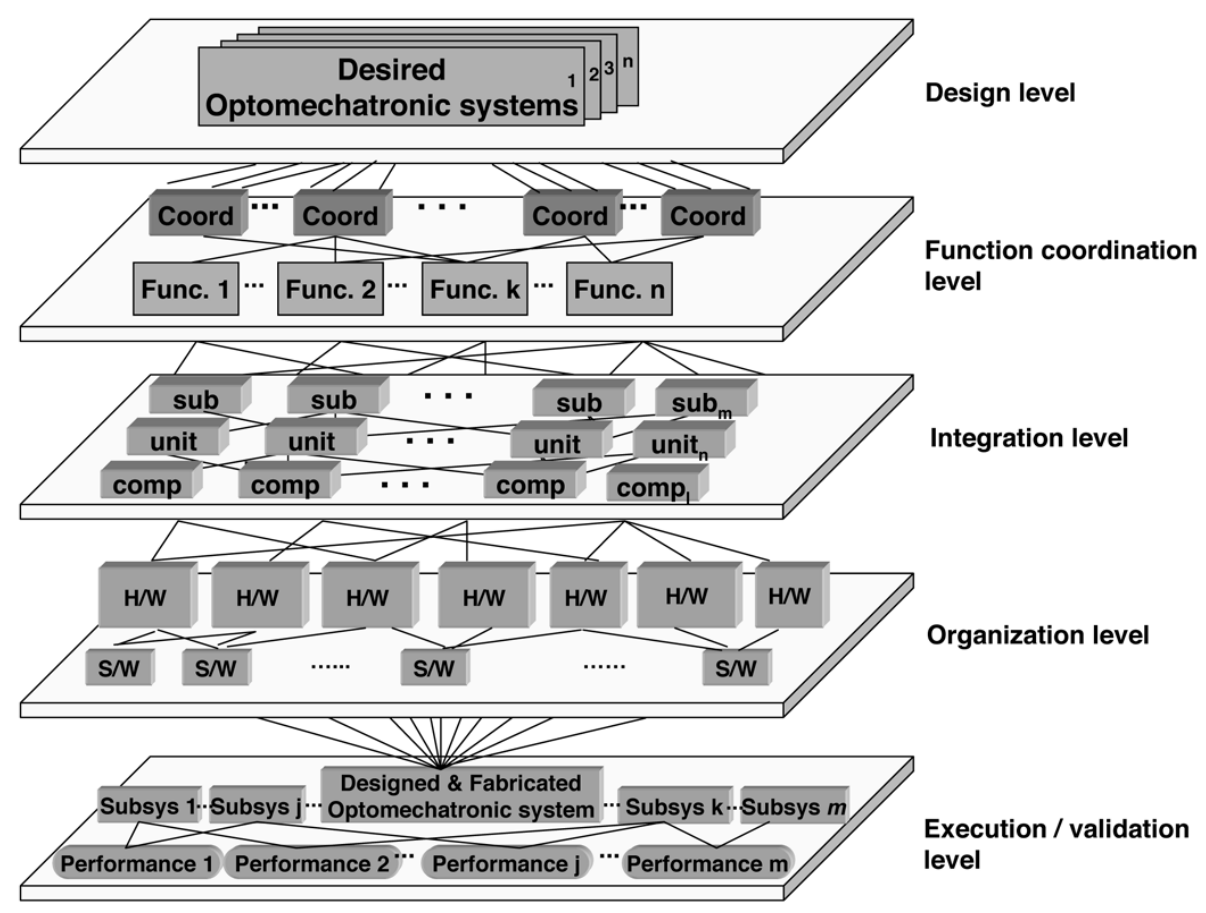

Fig. 8. Hierarchical design process for optomechatronic system.

As shown in the figure, each of the constituents can be represented by the following equations:

$$
\begin{aligned}
S Y S & =\left\{S U B_{i}\right\}, \quad \text { for } i=1,2, \ldots, m \\
S U B_{i} & =\left\{U N T_{j}^{(i)}\right\}, \quad \text { for } j=1,2, \ldots n_{i} \\
U N T_{j}^{(i)} & =\left\{C O M_{k}^{(i, j)}\right\}, \quad \text { for } k=1,2, \ldots h_{i, j} \\
C O M_{k}^{(i, j)} & =\left\{E L_{l}^{(i, j, k)}\right\}, \quad \text { for } l=1,2, \ldots p_{i, j, k}
\end{aligned}
$$

where \{\} denotes the set composed of the constituents in the parentheses. $m$ is the number of subsystems, $n_{i}$ is the number of units composing the $i$ th subsystem, $h_{i, j}$ is the number of components constituting the $j$ th unit that belong to the $i$ th subsystem, and $p_{i, j, k}$ is the number of elements composing the $k$ th component that belongs to the $j$ th unit in the $i$ th subsystem. In the notation, $U N T_{j}^{(i)}$, the upper subscript " $(i)$ " represents that this unit is related to the $i$ th subsystem. Similarly, in $C O M_{k}^{(i, j)}$, “ $(i, j)$ " denotes that this component is connected to the $j$ th unit and the $i$ th subsystem. " $(i, j, k)$ " of $E L_{l}^{(i, j, k)}$ indicates that this element contributes to the construction of the $k$ th component that belongs to the $j$ th unit in the $i$ th subsystem.

Each element belongs to one of the three types: pure mechanical $\left(E L_{M_{P}}\right)$, pure optical $\left(E L_{O_{P}}\right)$, and pure electronical $\left(E L_{E_{P}}\right)$ elements. As expressed in (1) and (2), when these basic elements are assembled together to constitute a component, the assembly is grouped into one of the six types: pure mechanical $\left(C O M_{M_{P}}\right)$, pure optical $\left(C O M_{O_{P}}\right)$, pure electronic $\left(C O M_{E_{P}}\right)$, mechatronic $\left(C O M_{M E}\right)$, optoelectronic $\left(C O M_{O E}\right)$, and optomechanic $\left(C O M_{O M}\right)$ components. On the other hand, the unit, subsystem, or system, which are the constituents in higher levels than the component level, has seven integration types. This is because, as represented in (3), in addition to the previously mentioned six types the optomechatronic nature becomes newly generated in the levels of unit, subsystem, or system. It is noted that the lowest level in which the constituent with optomechatronic nature can be produced is the unit level.

\section{Classification of Optomechatronic Systems (OPME)}

Mechatronic systems embedded with optical constituents $(M E O)$ basically exhibit the characteristics of mechatronic systems. Referring to (3) and (4), and Figs. 1 and 7, if a system meets the following conditions:

1) $S Y S \subset M E$ and $S Y S \not \subset O E M$;

2) $C O M_{k}^{(i, j)}, U N T_{j}^{(i)}$, or $S U B_{i} \in O$ for $i=1,2, \ldots \ldots, m$, $j=1,2, \ldots \ldots, n_{i}$, and $k=1,2, \ldots \ldots, h_{i, j}$ where $O=O_{p} \cup O M \cup O E$, then $S Y S \subset M E O$.

On the other hand, an optical system embedded with mechatronic constituents (OEM) exhibits the characteristics of optical systems. Similarly, if a system meets the following conditions:

1) $S Y S \subset O$ and $S Y S \not \subset M E O$;

2) $C O M_{k}^{(i, j)}, U N T_{j}^{(i)}$, or $S U B_{i} \in M E$ for $i=$ $1,2, \ldots \ldots, m, j=1,2, \ldots \ldots, n_{i}$, and $k=$ $1,2, \ldots \ldots, h_{i, j}$ where $M E=M \cap E$, then $S Y S \subset O E M$.

This indicates that a basically optomechatronic unit or subsystem also has two different integration characteristics.

\section{Design Consideration of Optomechatronic Systems}

Once a design objective is given, it is important to know what type of fundamental functions should be needed, how they are generated via optomechatronics, and what type of components, units, and subsystems need to be integrated to obtain the desired functions. In other words, when an optomechatronic system is required to perform a specified task, it needs to possess a set of fundamental functionalities, which can be produced by forming an appropriate structural architecture to build the system. Fig. 8 


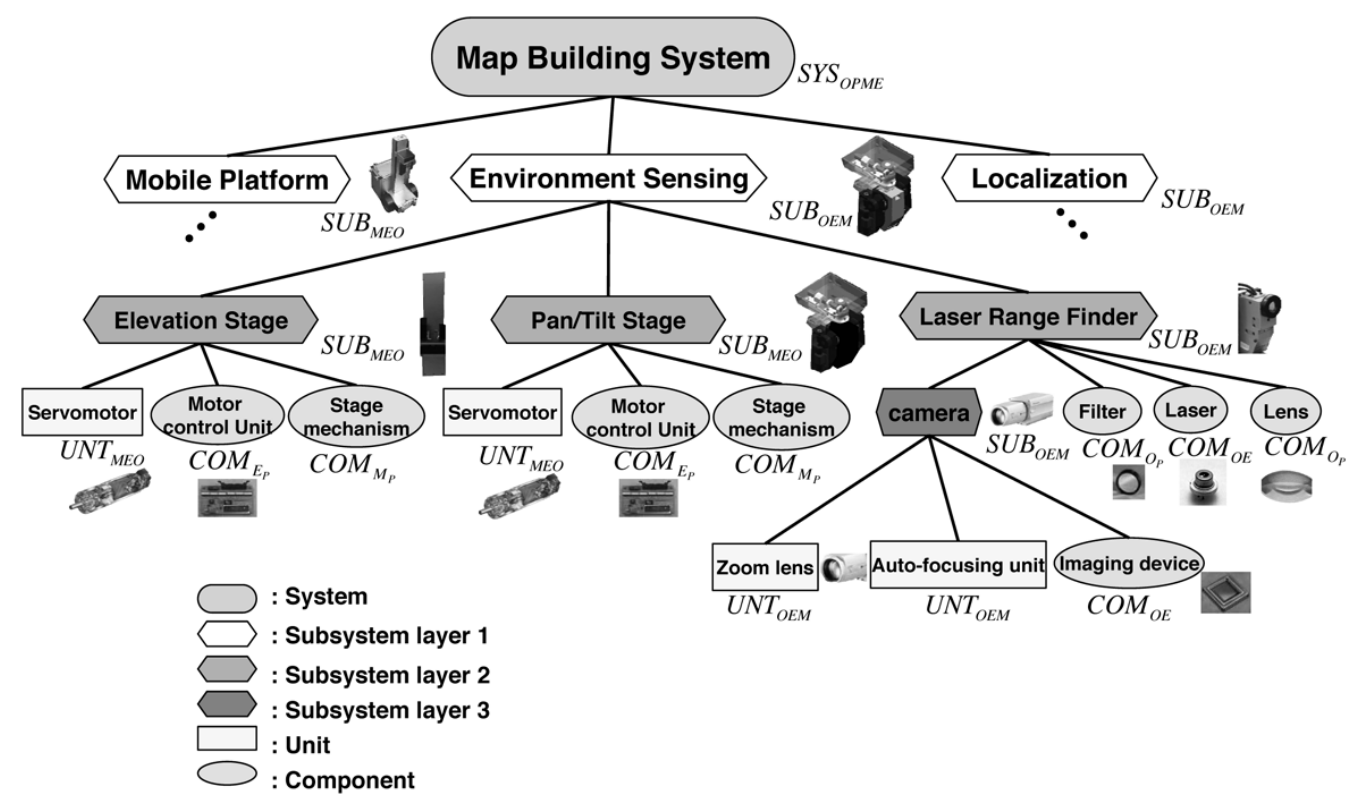

Fig. 9. System architecture of a mobile robot's map-building system.

depicts a generic hierarchical structure of integrating various functionalities needed to operate an optomechatronic system in a desired manner. The whole architecture is composed of four major levels: 1) conceptual system design level; 2) function coordination level; 3) integration level; 4) organization level of hardware and software; and 5) task execution and performance validation level.

Once a functional concept of a system is determined at the design level, the next step is to coordinate the functionalities needed to achieve the desired performance requirements at the function coordination level. With the coordinated functionalities, integration tasks need to be performed. After all integrations are properly made to form a desired system structure, the next step is to organize the configuration of real hardware $(\mathrm{H} / \mathrm{W})$ and software $(\mathrm{S} / \mathrm{W})$ based on the system functional requirements extracted at the functional coordination level. These are then combined to meet the desired integration at various levels of the system. Finally, at the task execution and performance validation level, the designed system is operated to execute tasks, and its performance is evaluated. If the system does not meet the required performance, the design process goes back to the integration stage and then repeats it again until system performance is met. The system shown in Fig. 9 illustrates well the above overall procedure of designing the three functionalities, mobility, sensing, and localization for map building.

To illustrate the above design procedure of an optomechatronic system, let us consider a mobile robot which contains a variety of optomechatronic components, units, and subsystems. One of the most important functionalities is to navigate autonomously in unknown space by acquiring the navigation environment information without human intervention. This environment perception system called a "map-building system" is interpreted as a typical optomechatronic system. Fig. 9 shows the system architecture of the map-building system composed of three subsystems [15]. According to the definition given in (1)-(4), it has three major optomechatronic subsys- tems: 1) "mobile platform" subsystem $\left(S U B_{M E O}\right)$ having the functionality of moving the robot; 2) "environment-sensing" subsystem $\left(S U B_{O E M}\right)$ having the functionality of sensing the surroundings; and 3) "localization" subsystem (SUBOEM) having the functionality of locating the robot during the navigation. As a representative example, let us analyze the "environment-sensing" subsystem from the viewpoint of the optomechatronic integration. This subsystem consists of three sub-subsystems in one level lower, second layer: 1) elevation stage $\left.\left(S U B_{M E O}\right) ; 2\right)$ pan/tilt stage $\left(S U B_{M E O}\right)$; and 3) laser range finder $\left(S U B_{O E M}\right)$. For example, the pan/tilt stage consists of a servomotor as a unit $\left(U N T_{M E O}\right)$, a motor controller as a pure electronic component $\left(C O M_{E p}\right)$, and a stage mechanism as a mechanical component $\left(C O M_{M p}\right)$. In particular, although the laser range finder is a subsystem in the second layer, it includes one subsystem in the third subsystem layer, which is the camera subsystem classified into $S U B_{O E M}$. This camera subsystem is composed of an optoelectronic component including CCD and electronic elements, and two units, a zoom lens unit $\left(U N T_{O E M}\right)$ and autofocusing unit $\left(U N T_{O E M}\right)$. Therefore, the map-building system can be identified as a system having the optomechatronic integration with which the three major functionalities are generated. It is noted that they are produced as a result of combination of functions generated by various optomechatronic elements within the system.

\section{Future Perspectives OF OPTOMEChATRONIC INTEGRATION}

In the previous sections, the nature of optomechatronic technology has been discussed and its fundamental integration characteristics have been identified. As we can anticipate from such features, optomechatronic integration is expected to take an important role in contributing to the advent of a new technological paradigm, since it can create unique functionalities that cannot be generated by mechatronic technology alone. The perspec- 
tives of the future direction led by this technology may be highlighted by the following aspects.

1) Creating new functionalities: System performance is often influenced by how many functionalities a system can possess to execute a variety of given tasks. Optomechatronic integration often creates new functionalities that mechatronics alone cannot provide due to some inherent characteristics that systems might have. This creation will enhance performance level measured by a variety of factors such as quality of system output, productivity, effectiveness, and cost.

2) Increasing the level of intelligence: If the level of intelligence is measured by such factors as flexibility, adaptability, and autonomy, optomechatronic technology will certainly enhance the level by fundamental functionalities as described previously.

3) Distributed characteristics: Most machines/systems have lumped characteristics in that they possess sensors, actuators, and controllers at discrete locations. It is, however, desirable for them to have distributed characteristics in order to obtain the necessary information which is often distributed spatially within them, and to generate necessary distributed action according to a distributed configuration. Embedding sensors, actuators, and controllers in a distributed manner into some delicate parts or structures of machine or system is a promising method to enable monitoring and control of their dynamic state.

4) Miniaturization: Due to the advances in technologies such as light-driven microfabrication processes, microoptics, microsensors and actuators, optomechatronic technology will be a prime moving technological source to drive miniaturization. Therefore, the system size, otherwise bulky and massive, will be drastically reduced, which in the future will bring about extensive use of optomechatronic components for the systems.

5) Integrated or embedded configuration: An integrated/embedded configuration in system structure is one of the salient characteristics of an optomechatronic system.These embedded characteristics, achievable with embedded sensors, controllers, and actuators, will facilitate multifunctional aggregation within a small unit and promote downsizing as well.

\section{CONCLUSION}

This paper has been focused on aiding the reader in understanding the optomechatronic technology in which optical, mechanical, and electrical/electronic engineering technologies are fused together. The definition and fundamental concepts of the technology were derived by analyzing the characteristics of a variety of practical optomechtronic systems currently being used. It was shown that optomechatronic technology can be generated by five basic types of integration of optical, mechanical, and electronic technologies. Further, fundamental functions were shown to be produced by varying the nature of integration. Based upon these, it was possible to identify how an optomechatronic system can be configured in order to create its desired functionalities. A detailed look into various optomechatronic systems currently being used manifests that optomechatronic technology is expected to make most engineered machine products and systems evolve toward having higher performance and intelligence in the future. In addition to this role, the technology will significantly contribute to the developments of: 1) micro/nanooptoelectromechanical systems; 2) biosystems which need nano/microsensing, control, and manipulation; and 3) micro/nanofabrication.

Optomechatronics will, therefore, become one of the core technologies that will dictate the future direction of various technologies.

\section{REFERENCES}

[1] K. Kincade, "Atomic-force microscopy finds new role in the nano world," Laser Focus World, vol. 40, no. 4, pp. 87-91, Apr. 2004.

[2] N. Wakami, H. Nomura, and S. Araki, "Fuzzy logic for home appliances," in Fuzzy Logic and Neural Networks, C. H. Chen, Ed. New York: McGraw- Hill, 1996, pp. 21.1-21.23.

[3] T. J. Krupa, "Optical R\&D in the army research laboratory," Opt. Photon. News, pp. 16-39, Jun. 2000.

[4] S. D. Robinson, "MEMS technology - micromachines enabling the all optical network," in Proc. Electronic Components and Technology Conf., Orlando, FL., Jun. 2001, pp. 423-428.

[5] K. Tsuruta, Y. Mikuriya, and Y. Ishikawa, "Micro sensor developments in Japan," Sens. Rev., vol. 19, pp. 37-42, 1999.

[6] T. V. Higgins, "Optical storage lights the multimedia future," Laser Focus World, vol. 31, no. 9, p. 103, Sep. 1995.

[7] S. G. Anderson, "Smart cars take the high-tech road," Laser Focus World, vol. 32, no. 6, p. 117, Jun. 1996.

[8] T. G. McDonald and L. A. Yoder, "Digital micromirror devices make projection displays," Laser Focus World, vol. 33, no. 8, p. s5, Aug. 1997.

[9] H. S. Cho, Opto-Mechatronic Systems Handbook: Techniques and Applications. Boca Raton, FL: CRC Press, 2003.

[10] D. A. Bradley, D. Dawson, N. C. Burd, and A. J. Loader, Mechatronics: Electronics in Products and Processes. London, U.K.: Chapman \& Hall, 1991.

[11] J. S. Kim and H. S. Cho, "A robust visual seam tracking system for robotic arc welding," Mechatronics, vol. 6, no. 2, pp. 141-163, 1996

[12] T. G. Lim and H. S. Cho, "Estimation of weld pool sizes in GMA welding process using neural networks," Proc. Inst. Mech. Eng., pt. I, vol. 207, no. 1, pp. 15-26, 1993.

[13] H. S. Cho, Optomechatronics: The Fusion of Optical and Mechatronic Engineering. Boca Raton, FL: CRC Press, 2005.

[14] H. Toshiyoshi, J. G. Su, J. LaCosse, and M. C. Wu, "Micromechanical lens scanners for fiber optic switches," in Proc. 3rd Int. Conf. Micro Opto Electro Mechanical Systems, Mainz, Germany, Aug. 1999, pp. 165-170.

[15] M. K. Kim, M. Y. Kim, and H. S. Cho, "A mobile robot navigation method based on sequential autonomous map building and self-localization using a laser vision sensor," in Proc. 8th Int. Conf. Rehabilitation Robotics, Daejeon, Korea, Apr. 2003, pp. 96-99.

[16] (2002-2005). Crown Equipment Co.-Image Gallery. [Online]. Available: http://www.crown.com/usa/products/html/2F.html

[17] (1995-2005). Samsung Electronics Co. [Online]. Available: http://www.samsung.com/

[18] Hefter Cleantech Co. [Online]. Available: http://www.hefter.de/ index.htm 


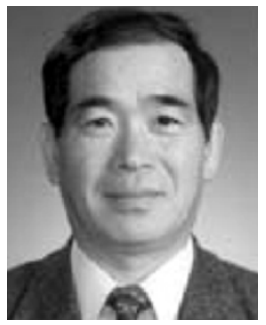

Hyungsuck Cho (M'94-SM'02) received the B.S. degree from Seoul National University, Seoul, Korea, in 1971, the M.S. degree from Northwestern University Evanston, IL, in 1973, and the Ph.D. degree from the University of California, Berkeley, in 1977.

Following a term as a Postdoctoral Fellow in the Department of Mechanical Engineering, University of California, Berkeley, he joined the Korea Advanced Institute of Science and Technology (KAIST), Taejon, Korea, in 1978. He has been a Visiting Professor at several universities, including the Institute für Produktionstechnik und Automatisierung (IPA), Ritsumeikan University, Japan, University of Paderborn, Germany, and New Jersey Institute of Technology. From 1995 to 1996, he was a Visiting Professor with the Advanced Manufacturing Program (AMP) of the University of California, San Diego. Recently, he was with the Department of Mechanical Engineering, University of Michigan. His research interests are focused on the area of optomechatronics, in particular, mobile robots, machine vision and pattern classification, and application of artificial intelligence/machine intelligence.

Prof. Cho serves on the Editorial Boards of the IEEE TRANSACTIONS ON InDUSTRIAL ElECTRONICS, Journal of Robotic Systems, Robotica, Control Engineering Practice, Journal of Advanced Robotics, and Journal of Engineering Manufacture. He also has been active in participating in and organizing international symposia/conferences. He has founded several international conferences, one of which is Opto-Mechatronic Systems of SPIE (ISAM), Optics-East (2000).

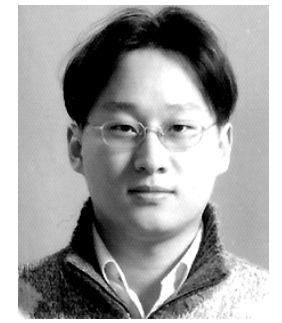

Min Young Kim received the B.S. degree in mechanical engineering and the M.S. and Ph.D. degrees from the Korea Advanced Institute of Science and Technology, Taejeon, Korea in 1996, 1998, and 2004, respectively.

From 2003 to 2004, he was a Researcher in the Intelligent Robot Research Center (ITRC-IRRC), Korea Advanced Institute of Science and Technology. Currently, he is a Research Engineer with Mirae Corporation, Cheonan, Korea, where he is involved in research work on artificial vision for machine and robot intelligence. His expertise is in the areas of sensor systems for robotic perception and 3-D map-building systems for robot navigation. He has studied a wide variety of robotic systems and their sensor systems, including wheeled service/industrial robots, and underwater robots.

Dr. Kim is a Member of The International Society for Optical Engineers and is serving as a Program Committee Member for IEEE ICRA 2005. 